Investigación

\title{
ASPECTOS BIOLÓGICOS Y CAPACIDAD DE DEPREDACIÓN DE Exochomus marginipennis (LeConte) (Coleoptera: Coccinellidae) SOBRE Diaphorina citri Kuwayama (Hemiptera: Liviidae)
}

\section{Research \\ BIOLOGICAL ASPECTS AND PREDATION CAPACITY OF Exochomus marginipennis (Leconte) (Coleoptera: Coccinellidae) ON Diaphorina citri Kuwayama (Hemiptera: Liviidae)}

Martín Palomares-Pérez ${ }^{1}$, Beatriz Rodríguez-Vélez ${ }^{1}$, Miguel Angel Ayala-Zermeño ${ }^{1 *}$, José de Jesús de la Cruz-Llanas ${ }^{1}$, Aimeé Monserrat Mendoza-Castañeda ${ }^{2}$, Jorge Antonio Sánchez-González ${ }^{1}$, Hugo César Arredondo-Bernal ${ }^{1}$, Esther Gisela Cordoba-Urtíz ${ }^{1}$

${ }^{1}$ Centro Nacional de Referencia de Control Biológico, SENASICA-DGSV Km 1.5 Carretera TecománEstación FFCC, Colonia Tepeyac, Colima, México. C.P. 28110.

2 Departamento de Parasitología Agrícola, Universidad Autónoma Chapingo. Km. 39.5 Carr. México Texcoco, Chapingo, Edo. de México C.P. 56230; aime.monserrat.19@hotmail.com

* Autor para correspondencia E-mail: ayalazermeno@yahoo.com.mx

\section{RESUMEN}

Se evaluó el ciclo biológico, tablas de vida y capacidad de consumo de Exochomus marginipennis (LeConte) sobre el psílido asiático de los cítricos Diaphorina citri Kuwayama, vector de la enfermedad Huanglongbing (HLB). El huevo tardó 5,83 $\pm 0,07$ días en eclosionar, mientras que la larva y la prepupa-pupa demoraron $16,4 \pm 0,73$ y $5,82 \pm 0,33$ días, respectivamente. La longevidad del adulto fue de $58,8 \pm 8,33$ días en promedio. La tasa de mortalidad específica $\left(\mathrm{q}_{\mathrm{x}}\right)$ mantuvo valores bajos en los cuatro estadios larvales y prepupa, durante el estado de huevo $\mathrm{q}_{\mathrm{x}}$ se incrementó, mientras que la fecundidad $\left(m_{x}\right)$ y la tasa neta de reproducción $\left(R_{0}\right)$ alcanzaron valores superiores a la unidad, evidenciando con esto un incremento en la población, a pesar de la alta mortalidad del estado de huevo. La larva IV $(98,8 \pm 2,61)$ seguida de la larva III $(97,60 \pm 3,43)$ y el adulto $(96 \pm 2.60)$ son los que ejercieron una mayor depredación. La ninfa $\mathrm{V}$ de $D$. citri es el instar menos consumido. Los resultados obtenidos exhiben por vez primera el ciclo biológico de E. marginipennis bajo condiciones controladas y soportan su capacidad de depredación sobre $D$. citri, para su potencial integración en programas de control biológico de esta plaga.

Palabras clave: Coccinellidae, psílido asiático de los cítricos, Candidatus Liberibacter spp., depredador, control biológico.

\section{ABSTRACT}

The life cycle, life tables and predation capacity of Exochomus marginipennis (LeConte) on the Asian citrus psyllid, Diaphorina citri Kuwayama, vector of Huanglongbing (HLB) was evaluated. The life time of $E$. marginipennis eggs was $5.83 \pm 0.07$ days, while the larva, pupa-prepupa life time was $16.4 \pm 0.73$ and $5.82 \pm 0.33$ days, respectively. Adult longevity was $58.77 \pm 8.33$ days on average. 
The specific mortality rate $\left(q_{x}\right)$ remained low in the four larval instars and prepupa. During the egg stage it $q_{x}$ increases, while fertility $\left(m_{x}\right)$ and net reproductive rate $\left(R_{o}\right)$ reached values greater than the unit, revealing with it an increase in the population, despite the high mortality of the egg. The larva IV $(98.77 \pm 2.61)$ followed by the larva III $(97.60 \pm 3.43)$ and the adult $(96.00 \pm 2.60)$ are the stages that are exerting a greater predation. Nymph $\mathrm{V}$ of $D$. citri is the instar consumed in smaller amounts. The results establish for the first time the life cycle of E. marginipennis under controlled conditions and its ability to sustain predation on $D$. citri. These results allow to decide on the use of E. marginipennis as a biological control agent.

Key words: Coccinellidae, Asian citrus psyllid, Candidatus Liberibacter spp., predator, biological control.

\section{INTRODUCCIÓN}

La familia Coccinellidae, con alrededor de 6000 especies reconocidas a nivel mundial (Zúñiga-Reinoso, 2011), tiene gran importancia ecológica y económica ya que tanto en la etapa adulta como larvaria son depredadores de insectos herbívoros (Obrycki y Kring, 1998). Entre las especies más importantes de esta familia encontramos a Olla v-nigrum (Mulsant), Cycloneda sanguinea L., Chilocorus cacti L., Axion sp., Arawana sp., Azya orbigera (Mulsant) y Brachiacantha decora (Casey) reportadas consumiendo pulgones, cóccidos y al psílido asiático de los cítricos (PAC) Diaphorina citri Kuwayama (Hemiptera: Liviidae) (Michaud, 2001, 2002, 2004; González et al., 2010), que es considerada la plaga más importante de los cítricos, vector de la bacteria limitada al floema Candidatus Liberibacter sp. (Martínez y Wallace, 1967) responsable de la enfermedad Huanglongbing (HLB) (Huang et al., 1984), causante de la muerte de millones de árboles en el mundo (Tsai et al., 2002; Halbert y Manjunath, 2004; Bové, 2006; Alemán et al., 2007).

En septiembre del 2013 se observó una especie de la familia Coccinellidae alimentándose de D. citri en árboles de naranja dulce (Citrus sinensis (L.) Osbeck) ubicados en la localidad de Los Asmoles, municipio de Colima, México (1906'19,76”' N y $103^{\circ} 46^{\prime} 35,99^{\prime \prime}$ W) y sobre plantas de Murraya paniculata (L.) Jack., rutácea considerada hospedante principal de D. citri (Hollis, 1985), localizadas en áreas circundantes a los invernaderos del Centro Nacional de Referencia de Control Biológico (CNRCB), en Tecomán, Colima (18 55'37,62”' N y $103^{\circ} 53^{\prime} 01,45^{\prime \prime}$ O). En mayo de 2014, el coccinélido fue identificado como Exochomus marginipennis (LeConte) (Coleoptera: Coccinellidae) y se estableció de igual forma su relación depredadora con D. citri (Palomares-Pérez et al., 2015).

Sin embargo en la literatura científica consultada no existe al momento información sobre aspectos biológicos y capacidad de consumo de esta especie sobre $D$. citri, por tal motivo se planteó como objetivo determinar el ciclo biológico y tablas de vida de E. marginipennis, así como su capacidad depredadora sobre el PAC con el pro- pósito de conocer su potencial como agente de control biológico de este vector.

\section{MATERIALES Y MÉTODOS}

El presente trabajo se llevó a cabo en el laboratorio de Insectos Entomófagos (IE) del CNRCB ubicado en la ciudad de Tecomán, Colima, México.

Se recolectaron 20 adultos de E. marginipennis sobre plantas de $M$. paniculata infestadas con $D$. citri, en los alrededores de los invernaderos del CNRCB empleando un aspirador bucal de 20 $\mathrm{mL}$. Los insectos se identificaron con las claves dicotómicas de Gordon (1985) y se encuentran resguardados en la Colección de Insectos Entomófagos (CIE) del CNRCB. Los adultos fueron sexados con base en la presencia de seis externitos visibles en la parte ventral de los machos, mientras que las hembras solo presentaron cinco (Gordon, 1985; Ślipiński, 2007). Se agruparon por parejas en recipientes de plástico de $25 \mathrm{~mL}$ con la tapa cubierta con tela de organza para que ovipositaran. Como sustrato de oviposición se colocó en el interior de los recipientes papel bond de 1 x 3,5 cm doblado en forma de acordeón. Los insectos se aparearon y su progenie se utilizó para iniciar los diferentes ensayos.

La alimentación tanto de las larvas como de los adultos se llevó a cabo con huevo de Sitotroga cerealella Olivier (Lepidoptera: Gelechidae). La investigación se realizó en condiciones controladas de $25 \pm 2{ }^{\circ} \mathrm{C}$, con una humedad relativa entre $60-70 \%$ y con un fotoperiodo de $14 \mathrm{~h}$ luz.

De igual forma, mediante muestreos mensuales de enero a diciembre del 2014 en los alrrededores del CNRCB, y con la ayuda de un estereoscopio, se registró la relación sexual hembra:macho mediante las características sexuales detalladas anteriormente.

\section{Ciclo biológico}

Para conocer el ciclo biológico, se colocaron 100 huevos de E. marginipennis en grupos de diez en cajas de Petri de $5 \mathrm{~cm}$ de diámetro revestidas con papel filtro humedecido para evitar el desecamiento y pérdida de la viabilidad. 
Para determinar el periodo de incubación, los huevos se mantuvieron en observación con la ayuda de un microscopio estereoscópico desde la puesta hasta su eclosión.

Una vez emergidas las larvas, se colocaron con la ayuda de un pincel de cerdas finas de forma individual en cajas de Petri nuevas. El periodo larvario se determinó con base a la presencia de la exuvia de cada uno de los estadios en desarrollo; una vez concluida la muda, la exuvia fue retirada con un pincel. Las larvas que alcanzaron el cuarto estadio fueron observadas para registrar el inicio de la prepupa y determinar la duración del estado de pupa. Al momento de obtener el adulto, se registró la fecha de emergencia para dar seguimiento a su longevidad.

Para los estados evaluados, la longevidad se definió como el tiempo de vida expresado en número de días promedio.

\section{Tablas de vida}

Las tablas de vida de E. marginipennis bajo las condiciones detalladas anteriormente se estudiaron en grupos de individuos (cohorte) nacidos dentro de un mismo intervalo de tiempo, desde el nacimiento hasta la muerte del último individuo (Rabinovich, 1980; Begon et al., 1988). Se utilizó una cohorte de 150 huevos, especificando para cada intervalo de edad los siguientes parámetros:

$\mathrm{X}=$ Edad en unidad de tiempo (días)

$\mathrm{N}_{\mathrm{x}}=$ Número total de individuos observados al inicio de cada estadio o estado

$1_{x}=$ Proporción de sobrevivientes al inicio de edad $\left(\mathrm{N}_{\mathrm{x}} / \mathrm{N}_{0}\right)$

$\mathrm{d}_{\mathrm{x}}=$ Número de muertos entre edades $1_{\mathrm{x}} \mathrm{y} \mathrm{l}_{\mathrm{x}}+1$

$\mathrm{q}_{\mathrm{x}}=$ Tasa de mortalidad $\left(\mathrm{d}_{\mathrm{x}} / \mathrm{l}_{\mathrm{x}}\right)$

$\mathrm{K}=$ Fuerza de mortalidad $\mathrm{K}=\log _{10} \mathrm{a}_{\mathrm{x}}-\log _{10} \mathrm{a}_{\mathrm{x}+1}$

$\mathrm{L}_{\mathrm{x}}=$ Individuos vivos entre $1_{\mathrm{x}} \mathrm{y} 1_{\mathrm{x}}+1$

$\mathrm{T}_{\mathrm{x}}=$ Tiempo que falta vivir hasta la extinción $(\Sigma$ inversa $1_{x}$ )

$\mathrm{e}_{\mathrm{x}}=$ Esperanza de vida $\left(\mathrm{T}_{\mathrm{x}} / 1_{\mathrm{x}}\right)$

$\mathrm{m}_{\mathrm{x}}=$ No. de hijas producidas por unidad de tiempo

$\mathrm{R}_{\mathrm{o}}=$ Tasa neta de reproducción $\sum_{\mathrm{x}} \mathrm{m}_{\mathrm{x}}$

\section{Capacidad de depredación}

Para comparar la capacidad de depredación de adultos de E. marginipennis y sus estadios larvarios, se colocaron 20 ejemplares de cada uno de los cinco estadios ninfales de $D$. citri en cajas de Petri de $5 \mathrm{~cm}$, las ninfas se pusieron sobre hojas de limón persa (Citrus aurantifolia (Christm) Swingle) recortadas circularmente, que sirvieron de soporte y alimento para las mismas. En cada caja de Petri con un conjunto de 100 ninfas (20 de cada estadio) se colocó por separado el adulto del coccinélido y cada uno de sus cuatro estadios, durante un periodo de $24 \mathrm{~h}$. Transcurrido el tiempo señalado, se contabilizó el número de ninfas consumidas. De igual forma, para investigar diferencias de depredación entre hembra y macho, se comparó la cantidad de individuos consumidos por cada sexo.

En este estudio también se evaluó la cantidad de huevos del PAC consumidos por los adultos y la larva I de E. marginipennis. Se recolectaron 200 adultos de $D$. citri en un vial de $20 \mathrm{~mL}$ de la cría del CNRCB. Con la ayuda de un estereoscopio se procedió a sexar a los individuos basados en el dimorfismo sexual de la especie; los machos son levemente más pequeños que las hembras y con la punta del abdomen romo, mientras que el abdomen de las hembras termina en punta bien marcada (Mead, 1977). Los adultos de D. citri se colocaron para que ovipositaran a razón de 20 individuos en una proporción similar de hembras y machos, durante un periodo de $48 \mathrm{~h}$ en 10 brotes tiernos y limpios de 5 a $10 \mathrm{~cm}$ de longitud de $M$. paniculata, Para evitar la fuga de los insectos, los brotes con los adultos se cubrieron con bolsas de tela de organza de $10 \times 5 \times 5 \mathrm{~cm}$. Transcurridas $48 \mathrm{~h}$, los adultos se sacrificaron, se retiró la tela de organza y se registró con la ayuda de un microscopio estereoscópico el número de huevos presentes en cada brote. Los brotes con los huevos fueron cortados con unas tijeras de podar y colocados durante $24 \mathrm{~h}$ en cajas de Petri de $5 \mathrm{~cm}$ con cada uno de los especímenes a evaluar.

Para uniformar los niveles de hambruna, los coccinélidos se mantuvieron en ayuno previo durante un periodo de $24 \mathrm{~h}$. Se realizaron 10 repeticiones y se colocaron en una cámara de cría con las condiciones ambientales antes señaladas.

Lo datos obtenidos se sometieron a un Análisis de Varianza (ANOVA) y separación de medias de Tukey al 5\% de confiabilidad con el paquete estadístico SAS 9.2 (2008). Para la capacidad de depredación entre hembras y machos se llevó a cabo una prueba t-student. En caso de no existir homogeneidad de varianza se utilizó un ajuste por raíz cuadrado.

\section{RESULTADOS}

\section{Ciclo biológico}

El tiempo de huevo a adulto de E. marginipennis fue de 28,05 $\pm 1,13$ días. La longevidad promedio del estado adulto fue de $58,77 \pm 8,33$ (Tabla 1 ) días con un valor máximo de 104 .

La duración del estado larvario fue de 16,40 \pm 0,73 días siendo la larva II y la larva IV los estadios de menor y mayor duración, respectivamente.

\section{Tablas de vida}

Los parámetros poblacionales registrados 
Tabla 1. Duración promedio (días) de los estados biológicos de E. marginipennis bajo condiciones de laboratorio $\left(25 \pm 2{ }^{\circ} \mathrm{C}, 60-70 \% \mathrm{HR}\right.$ y fotoperiodo de $14 \mathrm{~h}$ luz).

Table 1. Average duration (days) of biological states of E. marginipennis under laboratory conditions $\left(25 \pm 2^{\circ} \mathrm{C}, 60-70 \% \mathrm{RH}\right.$ and photoperiod of $14 \mathrm{~h}$ light).

\begin{tabular}{llr}
\hline Estado & & Días \pm D.E. \\
\hline Huevo & & $5,83 \pm 0,07$ \\
Larva & Larva I & $3,60 \pm 0,28$ \\
& Larva II & $3,20 \pm 0,14$ \\
& Larva III & $3,40 \pm 0,17$ \\
& Larva IV & $6,20 \pm 0,14$ \\
Prepupa & & $1,40 \pm 0,17$ \\
Pupa & & $4,42 \pm 0,16$ \\
\hline Adulto & & $58,77 \pm 8,33$ \\
\hline
\end{tabular}

en la tabla de cohorte de la población estudiada mostraron que la tasa de mortalidad específica $\left(q_{x}\right)$ mantiene valores bajos en los cuatro estadios larvales, prepupa y pupa. Esta condición difiere para el huevo, donde los valores de $\mathrm{q}_{\mathrm{x}}$ se incrementan (Tabla 2). La intensidad de la mortalidad (K) se comporta de igual forma, presenta valores bajos en los estadios larvarios, prepupa y pupa y altos para el estado de huevo.

En la misma Tabla 2 se aprecia que la esperanza de vida $\left(e_{x}\right)$ muestra un incremento notorio en la larva I, siendo por lo tanto el que presenta mayor expectativa de vida, a partir de éste empieza un descenso continuo hasta llegar a la prepupa, donde se presenta nuevamente un ligero incremento respecto a la larva IV.

De la población evaluada (cohorte), 21 individuos alcanzaron el estado adulto, representando un $14 \%$ de insectos que llegan a completar su ciclo, la fecundidad $\left(m_{x}\right)$ y la tasa neta de reproducción $\left(R_{\mathrm{o}}\right)$ alcanzaron valores superiores a la unidad.

En lo que se refiere a la relación sexual, los muestreos realizados mostraron un dominio de las hembras sobre los machos, el cual se manifes- tó por una relación hembra: macho de 1,5:1. Esta condición se repitió en la tabla de vida donde el dominio de la hembra manifestó una relación de igual forma 1,6:1.

\section{Capacidad de depredación}

El análisis estadístico con ajuste de raíz cuadrada indica una diferencia significativa al comparar la depredación que ejercen el adulto, y cada uno de los cuatro estadios larvales de E. marginipennis sobre los cinco estadios ninfales de $D$. citri. La comparación de medias de Tukey con una $\alpha$ $=0.05$ se utilizó para corroborar esta diferencia (Tabla 3).

En la Tabla 3 se observa que la larva IV seguida de la larva III y el adulto, son los que ejercen una mayor depredación. En la misma tabla se puede apreciar que la ninfa $V$ de $D$. citri es el estadio consumido en menor cantidad por el adulto y los cuatro estadios larvales.

Comparando los insectos ofrecidos respecto a los consumidos, la larva I con un promedio de 7,5 \pm 1 ninfas depredadas fue la que menor cantidad de individuos consumió.

Así también, con un promedio de $97 \pm 3,21$

Tabla 2. Tablas de vida horizontal de E. marginipennis bajo condiciones de laboratorio $\left(25 \pm 2^{\circ} \mathrm{C}, 60\right.$ $70 \%$ HR, 14 h luz) en el CNRCB, Tecomán, Colima, México.

Table 2. Horizontal life tables of E. marginipennis under laboratory conditions $\left(25 \pm 2^{\circ} \mathrm{C}, 60-70 \% \mathrm{RH}\right.$, of 14 hours photoperiod light) in CNRCB, Tecomán, Colima, Mexico.

\begin{tabular}{lrrrrrrrrrr}
\hline $\mathbf{x}$ & $\mathbf{n}_{\mathbf{x}}$ & $\mathbf{1}_{\mathbf{x}}$ & $\mathbf{d}_{\mathbf{x}}$ & $\mathbf{q}_{\mathbf{x}}$ & $\mathbf{K}$ & $\mathbf{L}_{\mathbf{x}}$ & $\mathbf{T}_{\mathbf{x}}$ & $\mathbf{e}_{\mathbf{x}}$ & $\mathbf{m}_{\mathbf{x}}$ & $\mathbf{R}_{\mathbf{o}}$ \\
\hline H & 150 & 1,5 & 108 & 0,720 & 0,552 & 171 & 365,5 & 2,137 & & \\
LI & 42 & 0,42 & 17 & 0,404 & 0,225 & 54,5 & 194,5 & 3,568 & & \\
LII & 25 & 0,25 & 2 & 0,080 & 0,036 & 36,5 & 140 & 3,835 & & \\
LIII & 23 & 0,23 & 0 & 0,000 & 0,000 & 34,5 & 103,5 & 2,971 & & \\
LIV & 23 & 0,23 & 0 & 0,000 & 0,060 & 34,5 & 69 & 1,971 & & \\
Pupa & 23 & 0,23 & 2 & 0,086 & 0,039 & 34,5 & 68,0 & 1,000 & & \\
Adulto & 21 & 0,21 & - & - & - & - & - & - & 13 & 2,73 \\
\hline
\end{tabular}


Tabla 3. Consumo promedio de E. marginipennis sobre los cinco estadios ninfales de $D$. citri bajo condiciones de laboratorio $\left(25 \pm 2{ }^{\circ} \mathrm{C}, 60-70 \% \mathrm{HR}, 14 \mathrm{~h}\right.$ luz $)$ en un tiempo de $24 \mathrm{~h}$.

Table 3. E. marginipennis average intake of five nymphal instars of $D$. citri under laboratory conditions $\left(25 \pm 2^{\circ} \mathrm{C}, 60-70 \% \mathrm{RH}\right.$ and 14 hours photoperiod light) in a period of 24 hours.

\begin{tabular}{lcccccc}
\hline Estadio & \multicolumn{7}{c}{ Ninfas consumidas (Media \pm D.E. ${ }^{\mathbf{~}}$ ) } \\
\cline { 2 - 7 } larval & \multicolumn{1}{c}{ I } & II & III & IV & V & Total \\
\hline I & $2,2 \pm 0,7 \mathrm{~b}$ & $3,0 \pm 1,3 \mathrm{~b}$ & $2,2 \pm 0,7 \mathrm{c}$ & $0,1 \pm 0,3 \mathrm{c}$ & $0,0 \pm 0,0 \mathrm{c}$ & $7,5 \pm 1,0 \mathrm{c}$ \\
II & $20,0 \pm 0,0 \mathrm{a}$ & $19,2 \pm 0,8 \mathrm{a}$ & $18,7 \pm 1,3 \mathrm{~b}$ & $16,7 \pm 2,5 \mathrm{~b}$ & $16,4 \pm 2,0 \mathrm{~b}$ & $91,2 \pm 6,7 \mathrm{~b}$ \\
III & $20,0 \pm 0,0 \mathrm{a}$ & $19,8 \pm 0,4 \mathrm{a}$ & $19,7 \pm 0,6 \mathrm{ab}$ & $19,0 \pm 1,2 \mathrm{a}$ & $19,1 \pm 1,1 \mathrm{a}$ & $97,6 \pm 3,4 \mathrm{a}$ \\
IV & $19,9 \pm 0,3 \mathrm{a}$ & $19,8 \pm 0,4 \mathrm{a}$ & $19,9 \pm 0,3 \mathrm{a}$ & $19,8 \pm 0,6 \mathrm{a}$ & $19,3 \pm 0,9 \mathrm{a}$ & $98,7 \pm 2,6 \mathrm{a}$ \\
Adulto & $19,7 \pm 1,1 \mathrm{a}$ & $19,4 \pm 0,9 \mathrm{a}$ & $19,7 \pm 0,7 \mathrm{ab}$ & $19,4 \pm 0,6 \mathrm{a}$ & $17,8 \pm 1,7 \mathrm{ab}$ & $96,0 \pm 2,6 \mathrm{a}$ \\
Pr $>$ F & 0,0001 & 0,0001 & 0,0001 & 0,0126 & 0,0037 & 0,0001 \\
F Value & 29,79 & 8,18 & 84,00 & 3,72 & 4,23 & 94,66 \\
\hline
\end{tabular}

${ }^{1}$ D.E.: Desviación estándar.

*Medias con la misma letra por columna son estadísticamente iguales (Tukey, $\alpha=0,05$ )

y $97,29 \pm 1,38$ ninfas consumidas en $24 \mathrm{~h}$ por las hembra y los machos, respectivamente; el análisis estadístico no registró diferencia en el consumo entre ambos sexos $(\mathrm{F}=5,43 ; \mathrm{Pr}=0,0589)$.

De un promedio de 104,90 $\pm 3,78$ huevos ofrecidos a larva I y $87,74 \pm 4,05$ al adulto, solo consumieron $41,70 \pm 2,13$ y $42,00 \pm 3,16$, respectivamente. El análisis estadístico reveló que no hay diferencia entre estos dos estados a la depredación de huevos ( $\mathrm{F}=0,92 ; \operatorname{Pr}>0,4156)$. Estos datos representan un porcentaje de depredación de $39,75 \%$ para la larva I y $48,5 \%$ para el adulto.

\section{DISCUSIÓN}

A la fecha no existen antecedentes del ciclo biológico de E. marginipennis, por lo cual los resultados del presente estudio se comparan con el ciclo biológico de otras especies del mismo género. Michaud y Olsen (2004) mencionan que el ciclo de vida varía dependiendo de las condiciones ambientales y la alimentación, como sucede con Exochomus childreni Mulsant, donde estos mismos autores indican que desde la postura de huevo hasta la preoviposición, el ciclo biológico de esta especie es de 60,8 \pm 1 ,96 días, mientras que Sengonca y Arnold (2003), sin mencionar la longevidad del adulto, reportan para E. quadripustulatus, un tiempo de desarrollo de larva a pupa de $28,3 \pm$ 1,8 días a una temperatura de $25^{\circ} \mathrm{C}$.

Durante el estado larval, E. marginipennis pasa por cuatro estadios, tal como ha sido registrado para la mayoría de las especies de Coccinellidae (Hodek, 1973; de Oliveira et al., 2004), siendo la larva II y la larva IV los estadios de menor y mayor duración, respectivamente. Machado (1982) señala que la mayor duración del $4^{\text {to }}$ instar está relacionada con la necesidad de incrementar los nutrientes requeridos para la transformación en pupa y posterior emergencia del adulto. Esta situación se asemeja a otras especies del mismo género como E. quadripustualtus y E. childreni, donde Muma (1956) y Sengonca y Arnold (2003) encontraron que el estadio IV es el más longevo.

Los parámetros poblacionales registrados en la tabla de cohorte de la población estudiada indican que las condiciones de alimentación, temperatura y humedad en la que se llevó a cabo el experimento son adecuadas para el desarrollo de la larva y la pupa; lo anterior no es válido para el huevo, donde los valores de mortalidad se incrementan.

Ésta observación se puede sustentar en la intensidad de la mortalidad (K) donde se aprecian valores bajos en los estadios larvarios, prepupa y pupa y altos para el estado de huevo. Michaud (2012) menciona que los huevos de los coccinélidos son el estado más frágil y por lo tanto están sujetos a una variedad de factores de mortandad.

A pesar de la alta mortalidad en el estado de huevo, la fecundidad $\left(\mathrm{m}_{\mathrm{x}}\right)$ y la tasa neta de reproducción $\left(R_{o}\right)$ alcanzaron valores superiores a la unidad, revelando con esto que las condiciones en las que se criaron los insectos propician un incremento en la población (Southwood, 1978; Rabinovich, 1980).

En lo que se refiere a los sexos, Doutt (1964) y Pfannenstiel y Yeargan (2002) mencionan que los depredadores tienen proporción sexual casi uniforme (50:50). Sin embargo, durante los muestreos realizados en campo, se registró que la proporción sexual de E. marginipennis mostró un predominio de las hembras sobre los machos, condición que se repitió en la tabla de vida, resultados que posiblemente indican una mayor presencia de la hembra sobre el macho bajo con- 
diciones naturales, y que la alimentación (presa) posiblemente no influye sobre la relación de sexos como sucede con otras especies de la familia Coccinelidae, como lo afirman Angulo et al. (2011).

Exochomus marginipennis presenta un tamaño pequeño que va de 2 a 3,60 mm (Gordon, 1985) circunstancia que le da la capacidad a D. citri de oponer mayor resistencia a la depredación en los estadios de mayor tamaño, siendo por lo tanto la ninfa $\mathrm{V}$ el instar consumido en menor cantidad tanto por el adulto como por los cuatro estadios larvales. Esta misma condición posiblemente sea la que explique el comportamiento de alimentación. El adulto y la larva II, III y IV consumen en su totalidad los estadios ninfales más pequeños de D. citri (I, II y III), mientras que las ninfas de mayor tamaño (IV y V) son consumidas parcialmente (Palomares-Pérez et al., 2015). La misma conducta de alimentación se presenta en la larva I, solo que ella come en su totalidad la ninfa I, mientras que la ninfa II y III solo son mutiladas, situación suficiente para impedir el desarrollo de D. citri.

Comparando los insectos ofrecidos respecto a los consumidos, la larva IV fue la que consumió mayor cantidad de individuos, a diferencia de la larva I que fue la que menor cantidad consumió. Ésto se puede explicar por la necesidad de la larva IV de guardar reservas para el periodo pupal (Omkar y Srivastava, 2001), mientras que la larva I probablemente debido a su tamaño, prefiere alimentarse de huevos, tal como se observó al comparar la depredación de huevos entre adulto y LI.

El análisis estadístico no registró diferencias entre el consumo de la hembra con respecto al macho. Estudios realizados sobre la alimentación de cinco especies de coccinélidos sobre ninfas de $D$. citri, por Michaud y Olsen (2004) aseveran que las hembras de tres especies son significativamente más voraces que los machos, y en las dos restantes incluida una del género Exochomus (E. childreni), no mostró diferencias. Rhamhalinghan (1987) citado por Aguilar et al. (2005) plantea en forma general, que el mayor consumo de alimento de las hembras sobre los machos es debido a que necesitan mayor cantidad de proteínas y nutrientes para la producción de huevos, condición que no se observó en éste estudio; en las investigaciones de Omkar y Srivastava (2003), mencionan que el consumo equivalente de presas por hembras y machos, pudo haber estado influenciado por fenómenos como el tiempo de exposición de la presa, tiempo de búsqueda y nivel de hambre de los depredadores.

La depredación de huevos por el adulto y el primer estadio larval no registró diferencia entre ellos, la oviposición natural de huevos colocados por las hembras de $D$. citri sobre brotes aún ce- rrados de $M$. paniculata como un mecanismo de alimentación y protección de $D$. citri (Tsai y Liu, 2000) es posiblemente el factor que intervino para observar un consumo similar de huevos por ambos estados. La morfología convexa de los adultos disuadió al insecto para acceder hacia los huevos ubicados en la parte más profunda del brote, por otro lado, las dimensiones corporales ( $1 \mathrm{~mm})$, y la forma campodeiforme de la larva I fueron los factores que propiciaron su acceso para alimentarse de los huevos de D. citri. González-Fernández et al. (2010) reportan para E. cubensis un porcentaje de depredación de huevos de $D$. citri con valores de 33,33 al $41,46 \%$ y para estadio ninfal I hasta de un $40 \%$.

En los Estados Unidos de Norte América (Florida, Kansas y Texas) han observado a E. marginipennis como un insecto muy activo en primavera y verano, sin embargo, asocian esta actividad a la presencia de pulgones y no con $D$. citri (Evans, 2014). Por otro lado, en Cuba se ha observado a miembros del género Exochomus (E. cubensis Dimmock), alimentándose de forma natural de $D$. citri (González et al., 2003). En estudios realizados en Florida, E.U.A., Michaud y Olsen (2004) determinaron varias especies de coccinélidos como depredadores muy voraces del PAC, entre ellos $E$. childreni.

\section{CONCLUSIÓN}

Los resultados obtenidos en el presente estudio establecen por vez primera el ciclo biológico de E. marginipennis bajo condiciones controladas, de igual forma sustentan su capacidad de depredación sobre $D$. citri, para considerar su posible inclusión en programas de control biológico del PAC.

\section{LITERATURA CITADA}

Aguilar A., D. Emmen, y D. Quiros. 2005. Respuesta funcional de Diomus sp. (Coleoptera: Coccinellidae) sobre Aphis craccivora (Homoptera: Aphididae). Tecnociencia 7(2):109-122.

Alemán, J., H. Baños, y J. Ravelo. 2007. Diaphorina citri y la enfermedad Huanglongbing: una combinación destructiva para la producción citrícola. Revista de Protección Vegetal 22(3):154-165.

Angulo, J., E. Arcaya, y R. González, 2011. Aspectos biológicos de Menochilus sexmaculatus (F) (Coleoptera: Coccinellidae) alimentado con Aphis craccivora Koch (Hemiptera: Aphididae). Boletín del Centro de Investigaciones Biológicas 45(4):423-432.

Begon, M., J.I. Harper, y C.R. Colin. 1988. Ecología: individuos, poblaciones y comunidades. Editorial Omega S.A, Barcelona, España. 
Bové, J.M. 2006. Huanglongbing: A destructive, newly emerging, century-old disease of citrus. Journal Plant Pathology 88:7-37.

Doutt, R.L. 1964. Biological characteristics of entomophagous adults. p. 145-167. In DeBach, P., and E. Schlinger (eds.). Biological Control of Insect Pests and Weeds. Reinhold Publishing Corporation, New York, USA.

Evans, A. V. 2014. Beetles of Eastern North America. Princeton University Press. 313 p. Disponible en http://books.google.com.mx/ books?id=zqVrAwAAQBAJ\&pg=PT533\&lp$\mathrm{g}=\mathrm{PT} 533 \& \mathrm{dq}=$ exochomus+marginipennis\&source=bl\&ots=ii1ExO1Ms3\&sig=kzxv3ZxLU_TSAFalhWp2ac2nKiU\&hl=es\&sa=X\&ei=gWokVPTtDYrAigLIvYG4Cg\&ved $=0$ C CcQ6AEwAjgU\# $\mathrm{v}=$ onepage \& $\mathrm{q}=$ exochomus\%20marginipennis\&f=true (Consulta 18 septiembre 2015).

González-Fernández, C., M. Borges, M. Gómez-Pacheco, M. Fernández, D. Hernández-Espinoza, y J. Rodríguez-Tapia. 2003. Manejo de Diaphorina citri Kuw. (Hemiptera: Psyllidae) en agroecosistemas citrícolas de Cuba. Instituto de Investigaciones en Fruticultura Tropical. La Habana, Cuba.

González-Fernández, C., M. Gómez-Pacheco, D. Hernández-Espinosa, y J. Rodríguez-Tapia. 2010. Entomófagos asociados a las plagas citrícolas, Lepidosaphes gloverii Packard (Hemiptera: Diaspididae), Phyllocnistis citrella Stainton (Lepidoptera: Gracillariidae) y $D i-$ aphorina citri (Kuwayama) (Hemiptera: Psyllidae) en naranjo Valencia. Centro Agrícola 37(4):75-81.

Gordon, R.D. 1985. The Coccinellidae (Coleoptera) of America North of Mexico. Journal of the New York Entomological Society 93(1):1912.

Halbert, S.E., and K.L. Manjunath. 2004. Asian citrus psyllids Diaphorina citri (Sternorrhyncha: Psyllidae) and greening disease in citrus: A literature review and assessment of risk in Florida. Florida Entomologist 87:330-353.

Hodek, I. 1973. Biology of Coccinellidae. Academia Publishing House of the Czechoslovak, Academy of Sciences, Prague, Czechoslovak.

Hollis, D. 1985. Parapsylla, a Gondwanan element in the psyllid fauna of southern Africa (Homoptera). Zoological Journal of the Linnaean Society 83:325-342.

Huang, C.H. M.Y. Tsai, and C.L. Wang. 1984. Transmission of citrus likubin by a psyllid, Diaphorina citri. Journal Agricultural Research China 33(1):15-72.
Machado, V.L.R. 1982. Morfologia e aspectos biológicos de Olla v-nigrum (Mulsant, 1866) e Cycloneda conjugata Mulsant, 1850 (Col., Coccinellidae) predadores de Psylla sp. (Homoptera: Psyllidae) em sibipiruna (Caesalpinia pelthophoroides Benth). En Funichello, M., L., Lúcia-Costa, O.J., Aguirre-Gil, and A.C., Busoli. 2012. Aspectos biológicos de Cycloneda sanguinea (Coleoptera: Coccinellidae) alimentadas con pulgones criados en algodón transgénico Bollgard I ${ }^{\circledR}$. Revista Colombiana de Entomología 38(1):156-161.

Martínez, A.L., and J.M., Wallace. 1967. Citrus leaf-mottle-yellows disease in the Philippines and transmission of the causal virus by a psyllid, Diaphorina citri. Plant Disease Reports 51:692-695.

Mead, F.W. 1977. The Asiatic citrus psyllid, Diaphorina citri Kuwayama (Homoptera: Psyllidae). Circular No. 180. 4 p. Florida Department of Agriculture and Consumer Services. Division of Plant Industry, Florida, USA.

Michaud, J.P. 2001. Numerical response of Olla v-nigrum (Coleoptera: Coccinellidae) to infestations of Asian citrus psyllid (Hemiptera: Psyllidae) in Florida. Florida Entomologist 84(4):608-612.

Michaud, J.P. 2002. Biological control of Asian citrus psyllid, Diaphorina citri (Hemiptera: Psyllidae) in Florida: a preliminary report. Entomological News 113:216-222.

Michaud, J.P. 2004. Natural mortality of Asian citrus psyllid (Homoptera: Psyllidae) in central Florida. Biological Control 29(2):260-269.

Michaud, J.P. 2012. Coccinellids in biological control. In Hodek. I., H.F., van Emden and A., Honěk. (eds.). Ecology and behavior of the ladybird beetles (Coccinellidae). Wiley-Blackwell, New York, USA.

Michaud, J.P., and L.E. Olsen. 2004. Suitability of Asian citrus psyllid, Diaphorina citri, as prey for ladybeetles. Biological Control 49:417431.

Muma, M.H. 1956. Life cycles of four species of ladybeetles. Florida Entomologist 39(3):115118.

Obrycki, J.J., and T.J. Kring, 1998. Predaceous Coccinellidae in biological control. Annual Review of Entomology 43:295-321.

de Oliveira, N.C., C.F. Wilcken, e C.A. de Matos. 2004. Ciclo biológico e predação de três espécies de coccinelídeos (Coleoptera: Coccinellidae) sobre o pulgão-gigante-dopinus, Cinara atlantica (Wilson) (Hemiptera: Aphididae). Revista Brasileira de Entomologia 48(4):529-533. 
Omkar, and S. Srivastava. 2001. Comparative predatory potential of a ladybird beetle Coccinella septempunctata Linn. on six prey species. Biology Memories 27(2):59-63.

Omkar, and S. Srivastava. 2003. Functional response of the seven spotted lady beetle, Coccinella septempunctata Linnaeus on the mustard aphid, Lipaphis erysimi (Kaltenbach). Insect Science Application 23(2):149-152.

Palomares-Pérez, M., J.M. Rodríguez-Vélez, B. Rodríguez-Vélez, A. Marín-Jarillo, J.A. Sánchez-González, and H.C. Arredondo-Bernal. 2015. First record and predatory activity of Exochomus marginipennis (LeConte) (Coleoptera: Coccinellidae) on Diaphorina citri Kuwayama (Hemiptera: Liviidae). Entomological News 125(3):194-198.

Pfannenstiel, R.S., and K.V. Yeargan. 2002. Identification and diel activity patterns of predators attacking Helicoverpa zea (Lepidoptera: Noctuideae) eggs in soybean and sweet corn. Environmental Entomology 31:232-241.

Rabinovich, J. 1980. Introducción a la Ecología de Poblaciones Animales. Editorial Continental S.A., México. 313 p.

SAS. 2008. SAS Users Guide: Statistics Version 9.02 for Windows. SAS Institute Inc., Cary, North Carolina, USA.
Sengonca, C., and C. Arnold. 2003. Development, predation and reproduction by Exochomus quadripustulatus L. (Coleoptera: Coccinellidae) as predator of Pulvinaria regalis Canard (Homoptera: Coccidae) and its coincidence with the prey in the field. Journal of Plant Diseases and Protection 110(3):250-262.

Ślipiński, A. 2007. Australian ladybird beetle (Coleoptera: Coccinellidae), their biology and classification. 12 p. Australian Biological Resources Study, Collingwood, Vic., Australia.

Southwood, T.R.E. 1978. Ecological methods: With particular reference to the study of insect populations. Chapman and Hall, Nueva York, USA.

Tsai, J.H., and Y.H. Liu. 2000. Biology of Diaphorina citri (Homoptera: Psyllidae) on four host plants. Journal of Economic Entomology 96(6):1721-1725.

Tsai, J., J. Wang, and Y.H. Liu. 2002. Seasonal abundance of the Asian citrus psyllid, Diaphorina citri (Homoptera: Psyllidae) in Southern Florida. Florida Entomologist 85(3):446451.

Zúñiga-Reinoso, A. 2011. Los coccinélidos (Coleoptera: Coccinellidae) de la región de Magallanes: nuevos registros y distribución regional. Anales Instituto Patagonia 39(1): 59-71. 